\title{
Effect of metformin on glycemic variability and glycemic control in patients with prediabetes.
}

\author{
Esperanza Martínez-Abundis ${ }^{*}$, Tonatiuh González-Heredia ${ }^{2}$, Diana M. Hernández-Corona², Manuel \\ González-Ortiz ${ }^{1}$
}

${ }^{1}$ Department of Physiology, Institute of Experimental and Clinical Therapeutics, Health Science University Campus, University of Guadalajara, Guadalajara, Mexico

${ }^{2}$ Department of Biomedical Sciences, Tonalá University Center, University of Guadalajara, Guadalajara, México

\begin{abstract}
Background: Researching the topic of glycemic variability (GV) has been very interesting due to the possible relationship that exists between this phenomenon and the development of micro- and macrovascular complications. The aim of this investigation was to evaluate the effect that metformin had on the $\mathbf{G V}$ in pre-diabetic patients.

Materials and methods: A randomized, double-blind, placebo-controlled clinical trial was carried out in 20 subjects, both male and female participants were chosen. These subjects where then divided into 2 groups; 10 subjects received metformin $(500 \mathrm{mg})$ while the remaining 10 subjects received a placebo, twice daily, before breakfast and dinner over the course of 90 days. Metabolic screening, which included glycated hemoglobin A1c, fasting plasma glucose (FPG), 2 h oral glucose tolerance test with $75 \mathrm{~g}$ of dextrose and GV [area under the curve of glucose, mean amplitude of glycemic excursion (MAGE)], were measured for all patients at baseline and at the end of the study. Wilcoxon signed-rank, and MannWhitney test were performed as part of the statistical analysis.

Results: Baseline characteristics of both groups were similar. There was a significant decrease of FPG levels in the metformin group as compared to placebo $(6.3 \pm 0.7 \mathrm{vs} .5 .6 \pm 1.0 \mathrm{mmol} / \mathrm{L}, \mathrm{p}=0.047)$. There were no significant differences in the $\mathrm{GV}$ parameters in either of the two study groups.

Conclusion: Pre-diabetic subjects treated with metformin for 3 months showed a significant decrease in FPG when compared to placebo.
\end{abstract}

Keywords: Metformin, Glycemic variability, Prediabetes, Continuous glucose monitoring.

Accepted on July 02, 2018

\section{Introduction}

Pre-diabetes is a condition which represents an increased risk of developing diabetes and cardiovascular disease [1] and has also been associated with atherosclerosis and increased arterial stiffness, which may eventually lead to elevated pulse pressure [2]. Most of the complications attributed to glucose concentration disturbances are not solely related to chronic hyperglycemia. Glycemic variability (GV), for example, may play an important role in microvascular complications seen in individuals with type 2 diabetes through an increase in oxidative stress [3]. Recently, studies investigating the dynamic fluctuation of glucose concentrations have demonstrated the importance of increased $\mathrm{GV}$, not only in individuals with diabetes but also in obese and pre-diabetic individuals. Therefor $\mathrm{GV}$ has been associated with the pathogenesis of diabetes and its related micro- and macrovascular complications [4].
Metformin is one of the first line treatments currently recommended to prevent or delay the appearance of type 2 diabetes [1-5]. Metformin has consistently demonstrated to have the strongest evidence base and most favorable long-term safety profile amongst any pharmacological therapies currently used for diabetes prevention.1 The Diabetes Prevention Program (DPP), a multicentric, randomized, placebo-controlled trial, showed that the risk of developing diabetes was reduced by up to $31 \%$ through metformin intervention in pre-diabetic individuals when compared to placebo [2].

However, to date, there are few clinical trials or investigations which are able to corroborate the potential health benefits brought about by the use of metformin for GV [6,7]. Therefore, the aim of this study was to evaluate the effect that metformin had on the GV and glycemic control in pre-diabetic patients. 


\section{Material and Methods}

\section{Study design}

A randomized, double-blind, placebo-controlled clinical trial was carried out.

\section{Participants}

A total of 20 adults of both genders were included in this study, with ages ranging from 30 to 60 years old. The diagnosed of pre-diabetes was established according to the American Diabetes Association (ADA) criteria, using $2 \mathrm{~h}$ post-load plasma glucose (2 h PG) concentrations between 7.8 and 11.0 $\mathrm{mmol} / \mathrm{L}$, and FPG levels between 5.6 and $6.9 \mathrm{mmol} / \mathrm{L}$, sedentary lifestyle, and body mass index (BMI) between 25 and $34.9 \mathrm{~kg} / \mathrm{m}^{2}$. All individuals were required to be nonsmokers and their body weight must have remained stable for at least 90 days prior to the study. Participants with previously diagnosed diabetes mellitus, hypertension, thyroid, renal or liver disease, or any other chronic disease were excluded from the study. All subjects must not have been taking any drug, supplement, neutraceuticals or have been on any special diet for at least 6 months prior to enrollment. Pregnant and/or breastfeeding women were excluded (Figure $1)$.

Flow Diagram

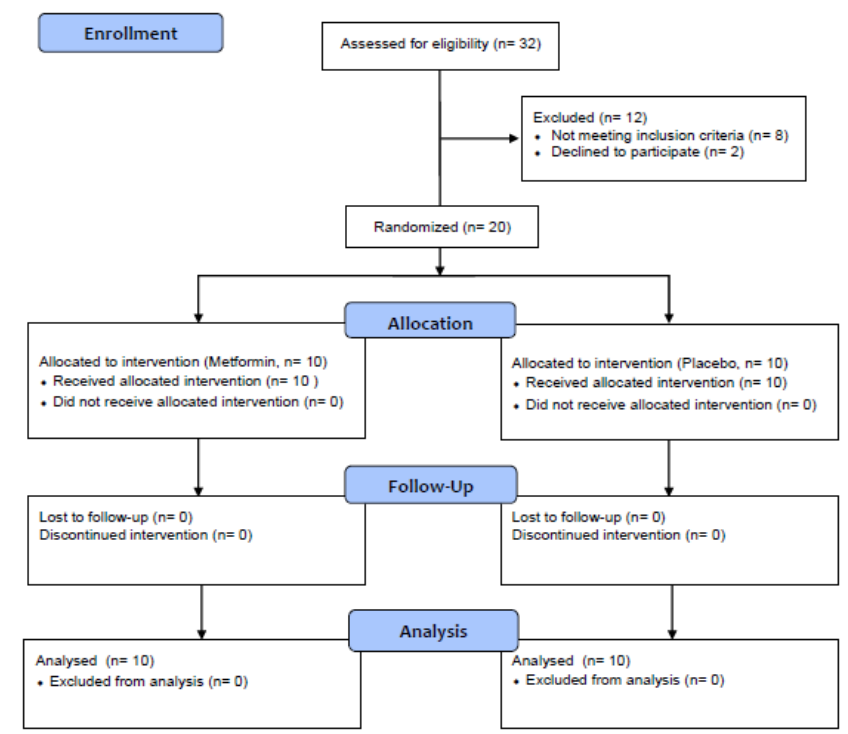

Figure 1. Flow chart of allocation of the study groups.

\section{Pharmacological administration}

After simple randomization using a computer generated random number list, the participants were allocated into one of two intervention groups of 10 subjects, each receiving either prolonged release metformin oral capsules $(500 \mathrm{mg}$ bid Predial plus, Laboratorios Silanes, S.A.de C.V., México) or placebo two times per day, once before breakfast and once before dinner, for 90 days. All subjects received recommendations regarding their medical nutrition therapy and were instructed to not modify their usual exercise habits. Clinical and laboratory measurements were evaluated at baseline and at 30,60 and 90 days. Tolerability and treatment compliance were assessed in all subjects at each of these periods.

\section{Procedures and calculations}

All enrolled subjects underwent an assessment prior to and after the intervention period. All tests were performed at 8:00 am after a 10-12 h overnight fast. Body weight was measured using a bioelectrical impedance digital scale and standing height was evaluated with the individual standing with their head aligned in the Frankfort horizontal plane. Both parameters were measured using a "Tanita" electronic scale, Model TBF-300 A (Tokyo, Japan). Waist circumference (WC) was measured at the midline between the highest point of the iliac crest and the lowest rib at the mid-axillary line with a certified, flexible, steel, Lufkin ${ }^{\circledR}$ tape measure. BMI was calculated as weight $(\mathrm{kg})$ divided by height squared $(\mathrm{m})$. Blood pressure was measured three times at the left arm with a digital sphygmomanometer (Omron Hem-907 XL®), with the subject in a seated position in a chair after 5 min rest. The mean of the three measurements was taken as the value for the systolic/ diastolic blood pressures and the measurements were expressed in millimeters of mercury ( $\mathrm{mmHg}$ ).

Blood samples were drawn from an antecubital vein after insertion of a catheter as an open route to facilitate the procedure and were drawn at baseline and at 30, 60, 90 and 120 min following a $75 \mathrm{~g}$ oral dextrose load, a procedure also known as oral glucose tolerance test (OGTT). Blood was centrifuged at $851 \mathrm{~g} \mathrm{(2500} \mathrm{rpm)} \mathrm{(Beckman} \mathrm{Coulter,} \mathrm{AllegraTM}$ $\mathrm{X}-22 \mathrm{R}()$ ) and plasma was collected in one aliquot for analysis to determine glucose and lipid profiles.

Glycemic control was defined by the assessment of fasting plasma glucose (FPG), 2 h PG and glycated hemoglobin A1c (A1C). Fasting plasma glucose (FPG) levels were measured by colorimetric methods using an automated analyzer (Erba XL-100®) with an intra and inter-assay coefficient of variation (CV) of $<1 \%$ and $2 \%$ respectively. A1C percentage was measured using ion-exchange high-performance liquid chromatography (Bio-Rad Laboratories, Hercules, CA, USA) with an intra- and inter-assay $\mathrm{CV}<2 \%$.

$\mathrm{GV}$ was assessed through the calculation of the area under the curve (AUC) of glucose, which was calculated with the polygonal formula, as well as by the mean amplitude of glycemic excursions (MAGE) and the standard deviation (SD) of glucose, through a continuous glucose monitoring system (CGMS) (GuardianReal Time ${ }^{\circledR}$; Medtronic Minimed, Northridge, CA). Continuous glucose monitoring (CGM) was conducted over a period of 3 days in both groups at baseline and after 3 months. For AUC of glucose, MAGE and SD of glucose values, we used the average of two consecutive measurements (on days 2 and 3 ) of the CGM. 
All subjects underwent clinical and laboratory evaluations beforehand, and were instructed to avoid strenuous physical activity at least $24 \mathrm{~h}$ before testing and to have a diet containing at least $250 \mathrm{~g}$ /day of carbohydrates during the 3 days prior to testing. Women were tested between days 3 to 8 of their menstrual cycle.

\section{Statistical analysis}

Sample size was calculated with a formula used for clinical trials for difference of means [8] with a statistical confidence level of $95 \%$ and a statistical power of $80 \%$, SD for glucose concentration of $1.0 \mathrm{mmol} / \mathrm{L} 6$ and MAGE [9], of $3.1 \mathrm{mmol} / \mathrm{L}$, and an expected difference between groups of at least 1.5 $\mathrm{mmol} / \mathrm{L}$ for glucose level and $4.4 \mathrm{mmol} / \mathrm{L}$ for MAGE, obtained from a total of 10 subjects per group accounting for an expected dropout rate of $20 \%$. For other components of GV and glycemic control, sample size calculation was equal or lower.

Data was analyzed using SPSS software (ver.21.0; SPSS, Inc., Chicago, IL, USA). Continuous variables are presented as means \pm SD and categorical variables as frequencies and percentages. Values are presented according to the International System of Units (SI). The Shapiro-Wilk test was used to evaluate normal distribution. Nonparametric statistics were used. After analysis, Wilcoxon signed-rank test and Mann-Whitney U-test were used to evaluate intra- and intergroup differences, respectively, and chi-square test was used to assess the differences in nominal variables. Intention to treat analysis was also performed. Subjects who dropped out were not replaced. The $\mathrm{p}$ value was considered significant at $\mathrm{p} \leq$ 0.05 .

\section{Ethical considerations}

The study protocol was reviewed and approved by the local ethics committee. The current study was performed in accordance with the ethical principles for medical research involving human subjects put forth by the international guidelines for Good Clinical Practices and the Declaration of Helsinki. All participants signed an informed consent form after being fully and accurately informed by the principal investigator regarding the nature, purpose, risks, and benefits of the study.

\section{Results}

A total of 20 pre-diabetic individuals were enrolled in the study, 10 per group (Figure 1), with an average age of $43.3 \pm$ 8.9 years in the placebo group and $48.3 \pm 6.8$ years in the metformin group $(\mathrm{p}=0.544)$. Mean BMI was $31.8 \pm 3.9$ and $31.4 \pm 3.3 \mathrm{~kg} / \mathrm{m}^{2}(\mathrm{p}=0.940)$, respectively. There were no significant differences in terms of clinical or laboratory parameters between the two groups at baseline (Table 1).

There were no significant differences in plasma A1C and $2 \mathrm{~h}-$ PG in patients taking placebo or metformin, neither was there any significant change in mean body weight in either group. FPG was significantly lower in patients taking metformin
( $p=0.047)$. The MAGE, AUC of glucose and SD of glucose showed no changes in either group after the intervention. Glycemic variability similarly showed no significant changes between the two groups (Figure 2).

Table 1. Clinical and laboratory characteristics of the study groups.

\begin{tabular}{lllll}
\hline & \multicolumn{2}{c}{ Placebo } & \multicolumn{2}{c}{ Metformin } \\
\cline { 2 - 5 } & Basal & Final & Basal & Final \\
\hline Weight $(\mathrm{kg})$ & $82.3 \pm 9.8$ & $83.3 \pm 10.9$ & $77.6 \pm 10.3$ & $77.2 \pm 10.6$ \\
\hline BMl $\left(\mathrm{kg} / \mathrm{m}^{2}\right)$ & $31.8 \pm 3.9$ & $31.8 \pm 3.9$ & $31.4 \pm 3.3$ & $31 \pm 3.5$ \\
\hline SBP $(\mathrm{mmHg})$ & $118.6 \pm 9.2$ & $119.5 \pm 12.3$ & $121.6 \pm 11.1$ & $117.6 \pm 6.3$ \\
\hline DBP $(\mathrm{mmHg})$ & $76.6 \pm 3.2$ & $76 \pm 13.2$ & $77.5 \pm 10$ & $74.4 \pm 4.8$ \\
\hline Glucose $(\mathrm{mmol} / \mathrm{L})$ & $6.5 \pm 0.5$ & $5.9 \pm 1$ & $6.3 \pm 0.7$ & $5.6 \pm 1.0$ \\
\hline Glucose at & $10.2 \pm 1.4$ & $10.1 \pm 2.9$ & $10.5 \pm 1.4$ & $10.3 \pm 3.3$ \\
$2 \mathrm{~h}(\mathrm{mmol} / \mathrm{L})$ & & & & \\
\hline A1C $(\%)$ & $5.8 \pm 0.4$ & $5.7 \pm 0.4$ & $5.7 \pm 0.4$ & $5.8 \pm 0.3$ \\
\hline MAGE (mmol/L) & $2.1 \pm 0.6$ & $1.8 \pm 0.5$ & $2 \pm 0.4$ & $1.7 \pm 0.4$ \\
\hline AUC of glucose & $153.2 \pm 17$ & $144.3 \pm 15.4$ & $146.7 \pm 8.1$ & $146.5 \pm 18$ \\
(mmol*h/L ) & & & & $0.8 \pm 0.2$ \\
\hline $\begin{array}{l}\text { SD of glucose } \\
\text { (mmol/L) }\end{array}$ & $0.8 \pm 0.4$ & $0.8 \pm 0.4$ & $0.6 \pm 0.3$ & \\
\hline BMI: Body Mass & & & & 0.8 \\
\hline
\end{tabular}

BMI: Body Mass Index; SBP: Systolic Blood Pressure; DBP: Diastolic Blood Pressure; A1C: Glycosylated Hemoglobin A1c; MAGE: Mean Amplitude Of Glycemic Excursion; AUC: Area Under The Curve; SD: Standard Deviation.

${ }^{*} p=0.047$, between basal and final values in the metformin group.

There were no significant differences in adverse events observed between groups. The main and most common side effect with metformin was an upset stomach $(n=1)$, while there were no side effects reported in the placebo group. No patient had to withdraw from the study because of adverse effects. Patient adherence to treatment was greater than $80 \%$.

\section{Discussion}

Recent studies have linked intra or inter day glucose fluctuations to an increase in the production of free radicals, a mechanism which may be involved in vascular endothelial damage. This investigation was carried out with the purpose of finding alternative uses for metformin in individuals with glucose intolerance, which could potentially modify these glycemic fluctuations that lead to high $\mathrm{GV}$, due to the fact that current evidence suggests that the chronic complications of type 2 diabetes begin to develop during the pre-diabetic state [10]. Likewise, it has also been noted that the risk of developing cardiovascular disease is two to three times higher in pre-diabetic individuals as compared to the general population [11], thus prevention of these complications becomes increasingly important. The ADA currently recommends the use of metformin in the prevention or delay of type 2 diabetes 1 , and they are just one amongst many other scientific sources that also support the use of metformin for 
prediabetes [12]. The current investigation showed that prediabetic individuals demonstrated a significant decrease in FPG when treated with metformin. This data coincides with a study carried out in a Chinese and Indian population, where it was shown that with a dose of $500 \mathrm{mg}$ of metformin twice a day, a significant reduction in fasting glucose levels could be achieved in a pre-diabetic population [13].
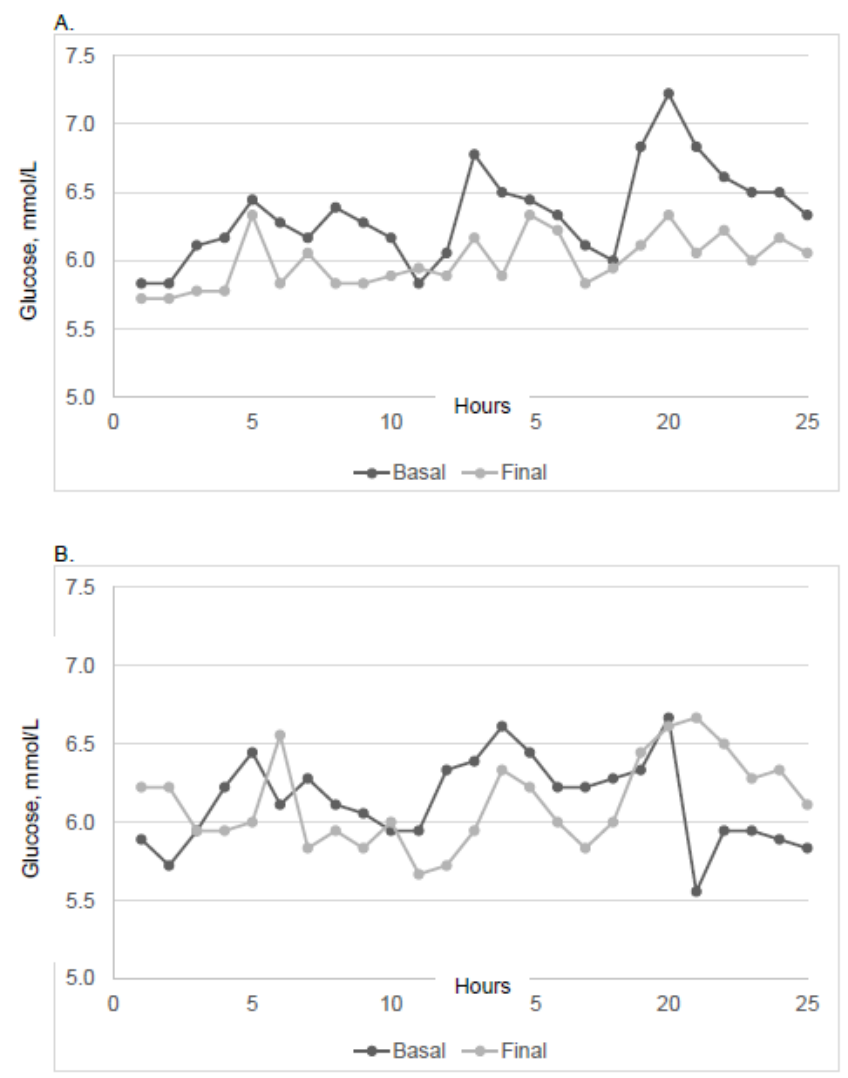

Figure 2. Glycemic variability behavior: metformin (A) and placebo (B) groups (basal vs. final). Glycemic variability showed a similar behavior in both groups.

Over the past few years, the study of daily glucose fluctuations and $\mathrm{GV}$ has been receiving increasing attention in regards to the important role that they play in the pathogenesis of the complications associated with diabetes via an increase in oxidative stress [14]. Recent studies have demonstrated that pre-diabetic individuals tend to have an increased GV and that this variability progresses and worsens in individuals with diabetes mellitus [15], especially in cases where abdominal obesity is a factor [16]. Unfortunately, there is currently little scientific evidence testing the utility of metformin in controlling GV in prediabetic patients. A similar study was found in which a significant decrease in GV was observed, however, the dose administered in the study was $850 \mathrm{mg}$ of metformin twice daily and diet and exercise parameters were controlled, factors which could have influenced the outcome of the study [17].

Despite the lack of a significant reduction in GV with the administration of metformin, it is important to remember that the acute and chronic behavior of glucose depends on a variety of different exogenous and endogenous components amongst which, elevated insulin resistance, elevated hepatic glucose production, increased production of counter regulatory hormones to insulin, sedentary lifestyle, poor feeding habits and age related metabolic deterioration, are key factors leading to the presence of events such as hyperglycemia, hypoglycemia and glucose fluctuations. However, even though no modification of $\mathrm{GV}$ was seen with the use of metformin, it continues to be one of the pillars in the treatment of these types of patients given the fact that, due to its mechanism of action and pharmacological effects, it continues to show the most evidence for the prevention or delay of type 2 diabetes mellitus $[3,18,19]$.

In terms of the presence of adverse events, no significant difference was found between the groups, only one adverse event was reported in a patient in the metformin group who presented gastrointestinal discomfort, the adverse event was slight, no concomitant medication was necessary for its resolution and it was not necessary to suspend the assigned treatment. This is consistent with the international literature, which shows that the rate of adverse effects is low (1-10\%) and that they are mostly gastrointestinal in nature, although, they can vary in frequency depending on the formulation and dose used. Despite this, there have been cases where the need to suspend treatment has been described, given that a certain percentage of patients fail to reach the total dose indicated due to gastrointestinal complaints [17].

In addition to the small sample size and the rigorous selection criteria implemented in this study, other possibly unidentified variables may have affected the outcome of this study such as: different eating patterns amongst the study population, insulin secretion and insulin resistance, and the exploratory nature of this study which produced limited results in the study population thus preventing their generalization.

In conclusion, pre-diabetic subjects taking metformin for 3 months showed improved glycemic control exhibited by a decrease in FPG when compared to placebo. Meanwhile, there was no significant difference in $\mathrm{GV}$ between the metformin and placebo groups.

\section{Acknowledgment}

We thank Assen Ognianov Iantchoulev, Executive Editor, Scientific Communications, for English editorial assistance.

\section{Conflicting Interests}

The authors declared the following potential conflicts of interest with respect to the research, authorship, and/or publications. This article was funded to the winner of the call of the Pfizer Scientific Institute Fund for Clinical Research 2013. From the Pfizer Mexico, S.A. of C.V. The funding agency had no role in the study design, data collection and analysis, decision to publish, or preparation of the manuscript. 


\section{References}

1. American Diabetes Association. Prevention or delay of type 2 diabetes. Diabetes Care 2018; 41: S51-S52.

2. Florez H, Temprosa MG, Orchard TJ, Mather KJ, Marcovina SM, Barret-Connor E, Horton E, Saudek C, PiSunyer XF, Ratner RE, Goldberg RB, Diabetes Prevention Program Research Group. Metabolic syndrome components and their response to lifestyle and metformin interventions are associated with differences in diabetes risk in persons with impaired glucose tolerance. Diabetes Obes Metab 2014; 16: 326-333.

3. Salkind SJ, Huizenga R, Fonda SJ, Walker MS, Vigersky RA. Glycemic variability in nondiabetic morbidly obese persons: results of an observational study and review of the literature. J Diabetes Sci Technol 2014; 8: 1042-1047.

4. Rodbard D. Clinical interpretation of indices of quality of glycemic control and glycemic variability. Postgrad Med 2011; 123: 107-118.

5. Vanita R, Aroda VR, Knowler WC, Crandall JP, Perreault L, Edelstein SL, Jeffries SL, Molitch ME, Sunyer XP, Darwin C, Heckman-Stoddard BM, Temprosa M, Kahn SE, Nathan DM, Diabetes Prevention Program Research Group. Metformin for diabetes prevention: insights gained from the Diabetes Prevention Program/ Diabetes Prevention Program Outcomes Study. Diabetologia 2017; 60: 1601-1611.

6. Pistrosch F, Köhler C, Schaper F, Landgraf W, Forst T, Hanefeld M. Effects of insulin glargine versus metformin on glycemic variability, microvascular and beta-cell function in early type 2 diabetes. Acta Diabetologica 2013; 50: 587-595.

7. Rodbard HM, Jellinger P, Davidson J, Einhorn D, Garber A, Grunberger G, Handelsman Y, Horton E, Lebovitz H, Levy P, Moghissi E, Schwartz S. Statement by an American Association of Clinical Endocrinologists/ American College of Endocrinology consensus panel on type 2 diabetes mellitus: an algorithm for glycemic control. Endocr Pract 2009; 15: 540-559.

8. Jeyaseelan L, Rao PS: Methods of determining sample sizes in clinical trials. Indian Pediatr 1989; 26: 115-121.

9. Irace C, Fiorentino R, Carallo C, Scavelli F, Gnasso A . Exenatide improves glycemic variability assessed by continuous glucose monitoring in subjects with type 2 diabetes. Diabetes Technol Ther 2011; 13: 1261-1263.

10. Abdul-Ghani MA, DeFronzo RA. Pathophysiology of prediabetes. Curr Diab Rep 2009; 9: 193-199.
11. Bonora E. Postprandial peaks as a risk factor for cardiovascular disease: epidemiological perspectives. Int J Clin Pract Suppl 2002; 129: 5-11.

12. Hostalek U, Gwilt M, Hildemann S. Therapeutic use of metformin in prediabetes and diabetes prevention. Drugs 2015; 75: 1071-1094.

13. Weber MB, Ranjani H, Staimez LR, Anjana RM, Ali MK, Narayan KM, Mohan V. The stepwise approach to diabetes prevention: Results from the D-CLIP randomized controlled trial. Diabetes Care 2016; 39: 1760-1767.

14. Service FJ. Glucose variability. Diabetes 2013; 62: 1398-1404.

15. Wang C, Lv L, Yang Y, Chen D, Liu G, Chen L, Song Y, He L, Li X, Tian H, Jia W, Ran X. Glucose fluctuations in subjects with normal glucose tolerance, impaired glucose regulation and newly diagnosed type 2 diabetes mellitus. Clin Endocrinol (Oxf) 2012; 76: 810-815.

16. Ma CM, Yin FZ, Wang R, Qin CM, Liu B, Lou DH, Lu Q. Glycemic variability in abdominally obese men with normal glucose tolerance as assessed by continuous glucose monitoring system. Obesity (Silver Spring) 2011; 19: 1616-1622.

17. Faerch K, Amadid H, Nielsen LB, Ried-Larsen M, Karstoft K, Persson F, Jørgensen ME. Protocol for a randomised controlled trial of the effect of dapagliflozin, metformin and exercise on glycaemic variability, body composition and cardiovascular risk in prediabetes (the PRE-D Trial). BMJ Open 2017; 7: e013802.

18. Clain J, Ramar K, Surani SR. Glucose control in critical care. World J Diabetes 2015; 6: 1082-1091.

19. Rena G, Hardie DG, Pearson ER. The mechanisms of action of metformin. Diabetologia 2017; 60: 1577-1585.

\section{*Correspondence to}

Esperanza Martínez-Abundis

Department of Physiology

Institute of Experimental and Clinical Therapeutics

Health Science University Campus

University of Guadalajara

Mexico 